\title{
Investigating The Relationship
}

Between Insomnia, Menopausal Syndrome and Depression and Exploring The Therapeutic Effects of Jieyu Pill

Hui Wang

Beijing University of Chinese Medicine

Xucan Hou

Beijing University of Chinese Medicine

Xuemei Tian

Beijing University of Chinese Medicine

Yun Wang ( $\square$ wangyun@bucm.edu.cn )

Beijing University of Chinese Medicine https://orcid.org/0000-0003-3117-8955

\section{Research}

Keywords: Jieyu pill, Insomnia, Menopausal syndrome, Depression, Influence relationship, Graph database

Posted Date: December 30th, 2020

DOI: https://doi.org/10.21203/rs.3.rs-136219/v1

License: (9) This work is licensed under a Creative Commons Attribution 4.0 International License.

Read Full License 


\section{Abstract}

Background: This paper explores the effects of insomnia and menopausal syndrome on the depressive process at the cellular and molecular levels, and investigates the mechanisms by which the commonly used antidepressant formula, Jieyu Pill (JYP), also has efficacy in insomnia and menopausal syndrome.

Methods: Neo4j was used to construct a graph database based on three diseases and mechanism of JYP; PPI data were used to analyze the molecular mechanism of effects of the diseases on depression process; the entity grammar system was used to resolve the mechanism of JYP in treatment of two diseases.

Results: A database of JYP and three disease charts was constructed. The relationship between insomnia and menopausal syndrome in terms of molecular mechanisms and cellular communication on the depressive process was obtained. The mechanism of JYP in insomnia and menopausal syndrome was obtained.

Conclusions: The results of this paper, which was conducted from the point of view of cellular communication and molecular mechanisms, show that insomnia and menopausal syndrome can have an impact on the course of depression. Therefore, synergistic treatment should be given to patients with depression associated with these two disorders. JYP is effective for all three disorders, can comprehensively regulate the organism and has high compliance, making it a clinical formula worth promoting.

\section{Introduction}

JYP is developed on the basis of Xiaoyao Powder and Ganmai Dazao Decoction, and is composed of 10 Chinese medicines: Bupleuri Radix, Angelicae Sinensis Radix, Paeoniae Radix Alba, Curcmae Radix, Poria, Lilii Bulbus, Albiziae Cortex, Glycyrrhizae Radix et Rhizome, Wheat and Jujubae Fructus. JYP has good therapeutic efficacy, mild adverse reactions, is not easy to produce medical origin of the disease and high compliance [1]. In recent years, it is quite widely used in clinical practice. Shen et al. [2] investigated the clinical efficacy, adverse effects, and safety of depression treatment with JYP and Maprotiline through randomized controlled trials. The results showed that the efficacy of the two drugs was comparable, but there were fewer adverse reactions and higher patient compliance in the JYP group, making it more suitable for clinical application. Yu Hongmin [3] conducted a completely randomized controlled trial of 100 patients with anxiety-related insomnia; the control group was given Mirtazapine tablets only, and the treatment group was given Mirtazapine tablets and JYP. Clinical observations showed that after 8 weeks of treatment, Pittsburgh Sleep Quality Index Scale scores decreased in both groups, with a more significant decrease and lower adverse effects in the treatment group. Qian Hong [4] observed the clinical efficacy of JYP in 64 cases of menopausal depression, among which 38 cases were cured, 22 cases improved and 4 cases did not recover, with a cure rate of $59.38 \%$ and a total effective rate of $93.75 \%$, which indicates that the effect of JYP treatment is remarkable. 
Insomnia, menopausal syndrome and depression are closely related. Firstly, insomnia and depression have a strong relationship, with studies showing that up to $90 \%$ of depressed patients report co-morbid problems with insomnia [5]. A synthesis of previous research shows that insomnia can be considered a symptom or natural consequence of depression [6], or it can be considered to occur prior to a depressive episode and can serve as an influential risk factor for the recurrence of depression (Pigeon and Perlis, 2007). In addition, studies have reported that many of the symptoms required to diagnose depression, such as fatigue, diminished volitional activity, depressed mood, hopelessness, and poor concentration, can be attributed to elevated levels of insomnia (Manber and Chambers, 2009; Pigeon and Perlis, 2007; Spielman and Anderson, 1999). Secondly, menopausal syndromes also often coincide with depression because menopausal women are at a higher risk of developing depression due to a variety of factors, including hormonal fluctuations or decreases, organic decline, and family and social pressures [7]. The neurotransmitter opioid peptide in the hypothalamus, adrenaline, dopamine, etc. are significantly associated with the occurrence of hot flashes, and serotonin has endocrine, cardiovascular, and affective regulatory functions, and altered levels of these neurotransmitters are also strongly associated with the development of depression. 5-Hydroxytryptamine has endocrine, cardiovascular, and emotional regulatory functions, and alterations in the levels of these neurotransmitters are also strongly associated with the development of depression. The current understanding lies mainly in the significant decrease of estrogen and progesterone in perimenopausal women and the resultant indirect interaction with hypothalamic-pituitary related axes, inflammatory factors, monoamine neurotransmitters and neurotrophic factors to induce the development of depression [8].

In order to investigate the effects of insomnia and menopausal syndrome on depression, this paper will be investigated from both a more macroscopic cellular communication perspective and a microscopic molecular mechanism perspective. Cell communication is when a message from one cell is transmitted through a medium to another cell and a corresponding response is produced. The communication relationships between cells are important for understanding disease development and drug action mechanism. Xu Xianxiang et al. [9] believe that the causes of many different human diseases can be attributed to a common mechanism, which is an abnormal cellular communication system, and that most drugs, whether single or multi-component, exert their effects by acting on cells, acting as signaling molecules. Cell communication provides the best modern scientific exegesis for multi-component Taditional Chinese Medicine (TCM) and their multi-targeted modes of action, and studying the integrated regulatory role of TCM from the perspective of cell communication is expected to truly reveal the essence of the effects of TCM. In this paper, protein interaction data will be used to analyze molecular mechanism, i.e., the relationship between genes associated with two diseases and those associated with depression.

In order to visualize the relationship between the effects of insomnia and menopausal syndrome on depression, this paper will construct a graph database based on the three disorders and the mechanism of JYP. Neo4j is recognized as the best graph database for mining inter-entity relationships based on the attribute graph model [10]. In recent years, an increasing number of scientific studies have been conducted using Neo4j in various fields of research. For example, Zhao Kai et al. [11] used Neo4j to 
construct a small knowledge map based on Guizhi decoction prescriptions from the Treatise of Febrile Diseases, and realized the retrieval, traversal, and navigation of the Guizhi decoction prescription of Prescriptions - Formulas - Medicines, laying a certain foundation for the identification and selection of prescriptions and the development of artificial intelligence-assisted prescribing systems in the future.In order to solve the problems of knowledge dispersion, incompleteness and inconvenience of querying in the process of handling cases in the judicial domain, Chen Jianxia et al. [12] extracted the semantic relations between entities and stored them in the form of ternary groups, and then imported the data into Neo4j graph database, thus realizing the construction, visualization and specific querying of the knowledge graph of judicial cases. Feng Li [13] uses Neo4j to construct a knowledge map of poetry in middle school languages, showing the structural relationship between poets and poems in the form of a diagram. Learners can look up the poem of a particular poet and browse the related poems and works, so that they can learn all the works of a particular author more systematically, thus consolidating and expanding their learning effect. Neo4j uses the Cypher language for data retrieval and query results; Cypher is a descriptive graphical query language that is relatively simple to use, but powerful enough to operate on graph databases without writing complex query code. It also supports the addition, deletion and correction of data [14]. In summary, Neo4j is widely used in various fields and is a very useful tool for storing, querying and visualizing data, so this paper uses Neo4j to fuse together a large amount of data collected for storage.

In this study, Neo4j will be used to construct a graph database based on three diseases and the mechanism of the JYP, and this paper investigates the influence of insomnia and menopausal syndrome on the depressive process from a more macroscopic cellular communication perspective and a microscopic molecular mechanism perspective, as well as the therapeutic effect of JYP on insomnia and menopausal syndrome.

\section{Materials And Methods}

\section{Data preparation}

\section{Disease-related genes}

This study collected disease-related genes for depression, insomnia, and menopausal syndrome from three databases. The OMIM database [15] (the Online Mendelian Inheritance in Man database, http://www.omim.org) is a comprehensive and authoritative compendium of human genes and genetic phenotypes, updated daily and free of charge, with entries containing a wealth of links to other genetic resources. The DrugBank database [16] (http://www.drug bank.ca, V5.1.4), a comprehensive, freely accessible, online database containing information on drugs and drug targets, combines detailed drug data with comprehensive drug target information, and almost all drugs listed in Wikipedia have their own DrugBank link. The TTD database [17] (Therapeutic Target Database, http://bidd.nus.edu.sg/group/cjttd/) is a database for providing information on known and explored therapeutic protein and nucleic acid targets, target diseases, pathway information, and corresponding 
drugs for these targets. TTD can be searched using drug, target, disease, etc. as keywords, with relevant literature attached to the results. Ultimately, 251 depression-related genes (DG) (Table S1), 140 menopausal syndrome-related genes (MPSG) (Table S2), and 115 insomnia-related genes (IG) (Table S3) were obtained by combining data and removing duplicate values.

\section{Chemical composition of JYP}

The TCMSP database [18] (Traditional Chinese Medicine Systems Pharmacology Database,http://Isp.nwu.edu.cn/tcmsp.php) and CASC database (Chinese Academy of Sciences Chemistry Database[http://www.organchem. csdb.cn) were respectively searched for the chemical composition of 10 TCMs in JYP. TCMSP provides the chemical composition of TCM contained in the pharmacopoeia and more comprehensive pharmacokinetic correlation data for drug screening and evaluation of each compound. It also provides potential target proteins for the compounds and proteinrelated diseases. It is beneficial to study the mechanism of TCM, discover the active ingredients of TCM, and optimize old prescriptions and new compound formulations from a systematic level [19]. CASC is an important part of the CASC scientific database, in which the plant chemical composition database can provide information on the chemical composition of herbs and natural products, it includes plant classification and picture information, isolated chemical composition and related research literature, etc. It integrates the plant species catalog, compound catalog and disease catalog, and collects 100,000 chemical substances, and supports many types of keyword searches, which is very convenient.

Each TCM contains numerous chemical compositions, but some of them cannot be effectively absorbed and utilized in the body, while others are not suitable for further development as drugs, therefore, two criteria have been set, namely, Oral Bioavailability $(O B) \geq 30 \%$ and Drug-Likeness $(D L) \geq 0.18$. This is to assess the characteristics of absorption, distribution, metabolism and excretion (ADME). Due to the multicomponent nature of TCM, different TCMs may contain one or more identical components, and after removing the duplicates, a total of 181 chemical compositions meeting the screening criteria were obtained (Table S4).

\section{Target proteins of chemical composition}

The efficacy of the drug and its effect on the organism depends on the extent to which the drug binds to the target protein and its disruption of the protein-protein and protein-compound interaction networks [20-22]. By the same token, the chemical compositions of TCM exert their effects through the same process. This study collected data on chemical composition-target proteins from the STITCH [20] (http://stitch.embl.de, V5.0) database. STITCH is a database providing known and predicted interactions between compounds and proteins, with data primarily derived from genomic background predictions, high-throughput experiments in the laboratory, co-expression (conserved), automated text mining, and prior knowledge of the database. It is widely used in the fields of virtual drug screening and analysis of mechanism. Since this thesis is about the effect of JYP on human body, the species code is restricted to "9606", and the file name of the downloaded data is 9606.protein_chemical. links.v5.0. tsv.gz. Some of 
the missing data were supplemented with data from the TCMSP database and data collected in the past by the subject group from databases and literature [23-25].

The following criteria were set based on the confidence level between the chemical composition and the target protein: for components with a high number of target proteins (10 or more), all entries with a confidence level of not less than 0.7 were selected if the number of entries was 10 or more. If there were fewer than 10 entries with a confidence level of at least 0.7 , the first 10 entries were selected to ensure data volume. For components with fewer target proteins (less than 10), all entries were selected; for data lacking scoring values, all entries were retained, as appropriate, as the number of targets per component was not particularly high. As a result, due to the lack of partial data, 19 chemical compositions were removed, resulting in 581 target proteins of 162 chemical compositions with a total of 1956 interactions between them (Table S5).

\section{Protein - protein interaction data}

Proteins and their functional interactions form the backbone of cellular mechanism, and consideration of the network of connections between them is necessary for a full understanding of biological phenomena. Protein-protein interaction (PPI) data in this study were obtained from the STRING [26] (https://string$\mathrm{db}$. org, V11.0) database. STRING is a database that provides known and predicted interactions between proteins and proteins, and is the most widely used database with the largest number of species and proteins, characterized by high coverage, ease of use, and having a consistent scoring system. This chapter restricts the species code to "9606" and the downloaded file to 9606.protein.links.v11.0.txt.gz, using PPI data with a confidence level of at least 0.4 and a clear activation or suppression relationship.

\section{Cell - cell communication data}

Data on inter-cellular communication were obtained from a paper examining multicellular signaling networks mediated by ligands and receptors [27] and from the HPA database [28] (The Human Protein Atlas, https://www.proteinatlas.org/). Data types include: cell types in the body, ligand and receptor genes on the cell surface, and other genes in the cell. The data in the literature are part of the FANTOM5 project, which focuses on the functional annotation of mammalian genomes and characterization of transcriptional regulatory networks, with RNA samples from major human organs, multiple progenitor cell types, over 200 cancer cell lines, 30 cell differentiation processes, and mouse developmental time courses sequenced using the Cap Analysis of Gene Expression (CAGE) method to facilitate further analysis. The HPA database aims to map all human proteins in cells, tissues, and organs using an integration of various histological techniques, using techniques including antibody-based imaging, mass spectrometrybased proteomics, transcriptomics, and systems biology.

\section{Building the graph database}

The data model of the Neo4j graph database consists of three main modules: nodes, attributes, and relationships. In this paper, the nodes of the graph database include the following: the JYP, 10 TCMs, chemical composition of TCMs, proteins (including target proteins of chemical composition, disease- 
related genes, PPI data, ligands and receptors on cells), and cell types. Relationships include: (JYP) -> [contain]-> (10 tradition Chinese medicines); (tradition Chinese medicines) $->$ [contain]-> (chemical composition); (chemical composition) $\rightarrow$ [act on]-> (protein); (protein A) $\rightarrow$ [activate/inhibit] $>$ (protein B); (cell) $>$ [ [express]-> (disease-associated gene); (cell) $>$ [ [express]-> (ligand); (cell) $->$ [express]-> (receptor); (ligand) $>$ [recognize binding]-> (receptor). The above nodes, relationships, and attributes are then stored in Neo4j as required by the Neo4j data import format.

\section{Analysis of the impact of insomnia and menopausal syndrome on depression}

In order to investigate the influence of insomnia and menopausal syndromes on the development of depression, this study will be carried out from both a macroscopic cellular communication perspective and a microscopic molecular mechanism perspective. On the one hand, querying cell communication data from two diseases to depression separately in a graph database using the match statement in the Cypher language. On the other hand, PPI data are used to analyze specific molecular mechanism, i.e., the role relationship between genes associated with the two diseases and those associated with depression.

\section{Analysis of the mechanism of JYP on insomnia and menopausal syndrome}

In order to obtain the mechanism of the chemical constituents of JYP on insomnia and menopausal syndrome, in this study, the mechanism will be analyzed using the methods and principles of the Entity Grammar System [29], as described in reference [30]. the Entity Grammar System is a formal grammar system suitable for modelling most complex systems. The Entity Grammar System is represented by a five-tuple: $G=(V N, V T, F, P, S)$. VN and VT are the basic elements; $F$ is the structural relationship of the basic elements; $P$ is the inference rule for known entities to infer new entities; $S$ is the initial state of inference, and $G=(V, F, P, S)$ if no distinction is made between VN and VT. The parsing results were then visualized as a network using Cytoscape.

\section{Results}

\section{Results of the construction of the graph database}

In order to visualize the functions that can be achieved with a graph database built on Neo4j, this study illustrates part of the mechanism of quercetin, a chemical constituent contained in Bupleuri Radix, as an example (Fig. 1).

In Fig. 1, the four individual circular nodes on the far left, from left to right, respectively represent the type of data: TCM, chemical composition of TCM, target of the chemical composition, and disease gene. The three yellow circular nodes to the right of the ESR2 gene represent the three cells that express this gene, the A2M gene can also be expressed in these three cells and can act as a ligand, while the LRP1 gene acts as a receptor that recognizes the A2M gene and binds to it for cell-to-cell signaling, and the 22 yellow 
circular nodes between the ligand and receptor genes represent the cells that can express them. Due to the limitation on the number of nodes that can be displayed, the graph is only a partial pass-through path, the full path is more complex and can be purposefully queried by entering different statements and adding restrictions as needed.

The graph database was constructed to facilitate the query of various information about the active ingredient groups, molecular mechanism and efficacy transfer between cells of JYP, which provides a systematic and efficient method and platform for the research of JYP.

\section{The impact of insomnia and menopausal syndrome on depression}

\section{Query results in the graph database}

The lookup path in the graph database can be summarized as follows: insomnia/menopausal syndrome - related genes - cell A expressing related genes - ligand on cell A - receptor - cell B expressing receptor depression related genes in cell $\mathrm{B}$. The match statement used is as follows:

Insomnia-Depression: match w=(:Disease\{Disease:"insomnia"\})-[:Related]-(:Gene)-[:Express]->(:C ell)[:Express]->(:Ligand)-[:Identification_and_combine]->(:Receptor)<-[:Express]-(:Cell)-[:Express]->(:Gene)[:Related]-(:Disease\{Disease:"depression"\})

return w limit 24

Menopausal syndrome - Depression: match w=(:Disease\{Disease:"menopausal syndrome"\})-[:Related] (:Gene)-[:Express]->(:Cell)-[:Express]]>(:Ligand)-[:Identification_and_combine]->(:Receptor)<-[:Express](:Cell)-[:Express]->(:Gene)-[:Related]-(:Disease\{Disease:"depression"\})

return w limit 24

Due to the large number of nodes and relationships involved, in order to clearly illustrate the relationship between the effects of the two diseases on depression, we limited the number of nodes that appear in the figure and set up and analyzed 24 cells involved in the effect relationship in both Fig. 2 and Fig. 3.

In Fig. 2, the red nodes represent the two diseases insomnia and depression, respectively; the purple node DRD1 is one of the insomnia-associated genes and MCHR1 is one of the depression-associated genes; the orange node represents the ligand, the blue node represents the receptor; and the green node is the cell expressing these genes. This figure contains some of the relationships of the effects of insomnia on depression, and the relationships would be even greater if the number of nodes present were not limited, suggesting that insomnia does have an effect on the development of depression.

In Fig. 3, the red nodes represent two diseases, menopausal syndrome and depression, respectively; the purple node HTR2A is one of the menopausal syndrome-associated genes, which is also a depression- 
associated gene, and MCHR1 is one of the depression-associated genes; the orange nodes represent ligands and the blue nodes represent receptors; and the green nodes are the cells expressing these genes. Likewise, this figure limits the number of nodes present and shows the relationship between the effects of menopausal syndrome on depression.

The above analysis shows that multiple relationships can be obtained using cell communication data to study the influence of two symptoms on the development of depression, and the numerous diseaserelated genes, ligands, receptors and cells involved in the study can be shown to have some influence on the development of depression.

\section{Results of analysis of molecular mechanism}

Using the disease-related gene data and PPI data collected during the construction of the graph database to find the effects of menopausal syndrome and IG on DG, 11 and 10 effect relationships were obtained respectively, and they are listed in Table 1.

Table 1

Relationship between menopausal syndrome genes, insomnia genes and depression genes

\begin{tabular}{|llllll|}
\hline MPSG & DG & FR & IG & DG & FR \\
\hline FMR1 & HRH1 & activation & CNR1 & HRH2 & activation \\
NR5A1 & TPH1 & inhibition & CNR1 & VPS13A & activation \\
BRD2 & ADRA2C & activation & PTGDR & TPH1 & activation \\
BRD2 & HRH2 & activation & SERPINA1 & HBA2 & activation \\
IL2 & TPH1 & activation & SIGMAR1 & PDGFB & activation \\
IL2 & BSN & activation & ADRA1B & PIP5K1C & activation \\
EIF2S2 & CPLX2 & inhibition & HTR7 & HBA2 & activation \\
AMH & SLC18A2 & activation & OPRM1 & BSN & activation \\
ADRA2C & LEP & activation & ADRA2C & LEP & activation \\
ADRA1B & PIP5K1C & activation & DRD1 & SMPD1 & activation \\
\hline DRD1 & SMPD1 & activation & & & \\
\hline
\end{tabular}

According to Table 1, the two disease-related genes are mostly activating for DG, i.e., upregulating their expression. $\mathrm{HRH} 1$ is a histamine $\mathrm{H} 1$ receptor that mediates neurotransmission in the central nervous system. the central histaminergic neuronal system regulates arousal, sleep-wake cycles, learning and memory, appetite control, and mood. HRH1 binding is reduced in the brains of depressed patients, and this reduction correlates with the severity of depressive symptoms [31]. TPH1 is tryptophan 5-hydroxylase 1 , which is involved in the metabolism of tryptophan in the presynaptic membrane and the formation of 
5-HT in the serotonergic synaptic signaling pathway, ensuring the production of sufficient amounts of 5$\mathrm{HT}$ required for organismal activity. ADRA2C is an $\mathrm{a}-2 \mathrm{C}$ adrenergic receptor that functions similarly to ADRA2B. It mediates catecholamine-induced inhibition of adenylyl cyclase, and binding to a-2 agonists inhibits the release of norepinephrine and acetylcholine. $\mathrm{HRH} 2$ is a histamine $\mathrm{H} 2$ receptor involved in the cAMP-PKA signaling pathway and mediates gastric acid secretion. In addition, it appears to regulate gastrointestinal motility, intestinal secretion, and cell growth and differentiation. $\mathrm{HRH} 2$ is associated with gastrointestinal function. BSN is bassoon protein, a scaffolding protein of the presynaptic cell matrix in the active region. The active region is the site of neurotransmitter release in the synapse. BSN participates in the PI3K-Akt signaling pathway, producing pro-apoptotic effects [32], and can also regulate presynaptic autophagy by interacting with $\mathrm{SIAH} 1$ to regulate specific presynaptic protein ubiquitination or by associating with ATG5. CPLX2, a complex element-2, negatively regulates the formation of synaptic vesicle aggregates from the active zone to the presynaptic membrane in postmitotic neurons, positively regulates the later steps of cytoplasmic vesicle cytomegaly in various cytoplasmic vesicles, and also participates in mast cell cytomegaly by similarity, which is related to neurotransmitter secretion and immune function. SLC18A2 is a synaptic vesicle amine transporter protein involved in ATP-dependent vesicle transport of biogenic amine neurotransmitters. It pumps cytoplasmic monoamines including DA, $\mathrm{NE}, 5-\mathrm{HT}$, and histamine into synaptic vesicles [33] to ensure proper neurotransmitter secretion. LEP is leptin, a key substance in regulating energy balance. In the hypothalamus, it is an appetite regulator and also regulates bone mass and hypothalamic-pituitary-adrenal hormone secretion [34]. It can influence the immune function of the body, modulate neutrophil activity and function, increase phagocytosis via macrophages, enhance the secretion of pro-inflammatory mediators, and increase the cytotoxic capacity of NK cells [35]. PIP5K1C is a phosphatidylinositol 4-phosphate 5-kinase type $1 \mathrm{\gamma}$, which is required for synaptic vesicle transport. It controls the plasma membrane pool of phosphatidylinositol 4,5bisphosphate associated with synaptic vesicle endocytosis and cytokinesis [36]. SMPD1 is a sphingomyelin phosphodiesterase that converts sphingomyelin to ceramide. It plays an important role in cell adhesion, promoting apoptosis and regulating cellular immunity. PDGFB is a platelet-derived growth factor subunit B. Growth factors are important in the regulation of embryonic development, cell proliferation, cell migration, survival, and chemotaxis. In summary, the biological processes involved in the DG in action are mainly focused on the transmission and secretion of neurotransmitters, with a predominant effect on synapses. These processes can also affect the immune function and inflammatory response of the body, as well as apoptosis and normal activity of cells, and all of them are closely related to the process of depression.

Cell communication data illustrate the more macroscopic link between disease and illness, which may not be directly linked, but the influencing relationships are present, and numerous relationships can be found when the data are queried. In contrast, molecular mechanism focus on genes and gene activation or repression relationships, with limitations, and embody fewer disease-to-disease relationships, but both are specific mechanism of action, with advantages and disadvantages for both. This also indicates that it is inadequate to understand the pharmacological mechanism of TCM only from the molecular mechanism, which can only show part of the mechanism and may ignore some important relationships. 
From the perspective of TCM theory, it is necessary to use holistic thinking to view problems from a systemic perspective. The mechanism study using cell communication data in this study can better reflect the dynamic process of activity transfer in TCM, and combine it with molecular mechanism for systematic analysis, which is in line with the idea of systematic Chinese medicine. The results of the combined cell communication data and molecular mechanism analysis show that insomnia and menopausal syndrome do have an influence on the development of depression, and therefore the treatment of these two symptoms will also be beneficial to the control and treatment of depression. This is especially true for patients who have a combination of depression and insomnia, or a combination of depression and menopausal syndrome. Since the presence of insomnia and menopausal syndrome symptoms not only increases the rate of misdiagnosis, but also may aggravate depression and cause relapse of the disease, simultaneous treatment of the disease will theoretically increase the efficiency of treatment and will also help to control the process and relapse of depression.

\section{Mechanism of JYP on insomnia and menopausal syndrome}

Insomnia and MPSG and relationships of action were obtained by the automatic reasoning function of the Entity Grammar System to understand the role of chemical compositions of JYP, and the nodes and relationships were visualized using Cytoscape (Fig. S1 and Fig. S2). Both figures include the direct and indirect effects of the chemical constituents on disease-related genes. There are 143 chemicals acting on 61 IG and 146 chemicals acting on 84 MPSG.

The pink nodes in Fig. S1 represent IG (A) and MPSG (B). The blue nodes in both figures represent the chemical composition of JYP, and the labels show the ID of the chemical composition, and the corresponding ingredient names are shown in Table S6. Using the above data on the role of the chemical constituents of JYP on insomnia and MPSG, as well as the role of DG on insomnia and MPSG in Table 1, we constructed a pathway by which JYP could exert antidepressant effects by intervening in both disorders (Fig. S2). The blue nodes in Fig. S2 represent the chemical composition of JYP, and the pink nodes are the IG and MPSG that have an effect on DG, including the ADRA2C gene, which is also a DG, and the orange nodes represent ligands.

From the above results, we can see that JYP also have a therapeutic effect on the two symptoms of insomnia and menopausal syndrome, and the previous section pointed out that they have an influence on the development of depression, and from this perspective, the relief of these two symptoms will also play an auxiliary role in the treatment of depression.

\section{Discussion}

One of the mechanisms of JYP in the treatment of depression is to achieve therapeutic effects by affecting insomnia and menopausal syndrome. The results of the mechanism JYP provide a new strategy for the treatment of depression, which is to reduce the risk of depression by alleviating insomnia and menopausal syndrome. The research results of pharmacological mechanism of TCM in recent decades have mainly focused on the elucidation of components and targets, but little research has been 
done on cellular communication. Unlike traditional pharmacological mechanism studies, this study not only analyzed the molecular mechanism of the antidepressant effect of JYP, but also analyzed the correlation between the antidepressant activity of the active ingredients in JYP formula and the antiinsomnia and anti-menopausal syndrome activities at the cellular communication level from the perspective of cellular communication, improved the existing mechanism analysis method, and integrated it into the Neo4j graph database, providing a systematic and efficient method and platform for the analysis of the effective ingredients in JYP. A systematic and efficient method and platform for the analysis of TCM herbal formulas was developed to systematically explore the influence of insomnia and menopausal syndrome on the process of depression, providing a new approach to the study of the relationship between insomnia, menopausal syndrome and depression, and exploring the potential of JYP for the treatment of insomnia and menopausal syndrome. Based on this, it is conducive to innovative ideas in the treatment of depression. The main two aspects can be as follows: (1) depression is a pathological state of the psychiatric system and somatization symptoms such as insomnia and menopausal syndrome have a causal and mutually reinforcing disease relationship, and the treatment strategy should consider simultaneous treatment in order to block the continuous development of depression; (2) depression is a systemic disease that involves many different cell types and cell functions, and should be treated on the basis of a systematic analysis of the relevant cell The therapeutic approach should be designed based on a systematic analysis of the relationship between the relevant cells.

However, the mechanism study at the level of cellular communication proposed in this paper reflects the non-directive influence relationship between diseases, which is still insufficient in the mechanism study of herbal prescriptions and needs to be further explored. Also, this paper only provides a study of the effects of insomnia and menopausal syndrome on the course of depression, which are only some of the somatization symptoms of depression. Other somatization symptoms of depression such as cognitive impairment, fatigue, immune deficiency, and pain can be investigated in future studies, and the graph database can be extended by adding genes related to other somatization symptoms to make it applicable to analyze the one-way or two-way influence relationship between somatization symptoms and depression. In addition, multiple prescriptions and multiple diseases can be further added and also analyzed using the methods provided in this study to achieve a bulk mechanism analysis of prescriptions acting on diseases, or to analyze the mechanism of alleviating multiple diseases for the same prescription to explore the potential of prescriptions to treat other diseases. With the continuous development of related fields and improvement of databases, the research methods combined with this paper can explore more mechanisms of prescriptions for treating diseases and provide a more efficient and objective technical system. The use of biological principles to analyze the mechanism of action of TCM is not the ultimate goal, but the analysis of the mechanism of action of TCM herbal formulas is of more practical value for biomedicine to draw on the wisdom of TCM to provide new ideas and strategies for disease treatment.

\section{Conclusions}


This study first provided basic data and a platform for subsequent relationship studies by constructing a graphical database based on the three diseases and the mechanism of JYP. Subsequently, cell communication data were used to query the pathways through which insomnia and menopausal syndrome affect depression, respectively, and PPI data were used to analyze the specific molecular mechanism involved, leading to the conclusion that the two diseases can affect the development of depression through multiple pathways. Finally, using the entity grammatical system to analyze the mechanism of the action of JYP on insomnia and menopausal syndrome, as well as the mechanism by which JYP acts as an adjunctive antidepressant while treating both symptoms, we will explore the potential of JYP in treating other diseases besides depression and expand the application of JYP.

The relationship between insomnia and depression, and between menopausal syndrome and depression, is complex, and a single treatment for only one of these disorders is difficult to cure and prone to relapse, as the presence of these symptoms can facilitate the depressive process. It has a therapeutic effect on depression, insomnia and menopausal syndrome, and it also has an antidepressant effect in the treatment of insomnia and menopausal syndrome. JYP can comprehensively regulate the body's neurotransmitter transmission and secretion, synaptic function, immune function and inflammatory response, which is more advantageous than a single drug for treating a particular disease, making it a clinically effective formula worthy of wide promotion.

\section{Abbreviations}

JYP: Jieyu Pill; TCM: Taditional Chinese Medicine; OMIM: Online Mendelian Inheritance in Man; TTD: Therapeutic Target Database; TCMSP: Traditional Chinese Medicine Systems Pharmacology; CASC: Chinese Academy of Sciences Chemistry; OB: Oral Bioavailability; DL: Drug-Likeness; ADME: absorption, distribution, metabolism and excretion; PPI: Protein-protein interaction; HPA: Human Protein Atlas; CAGE: Cap Analysis of Gene Expression; MPSG: menopausal syndrome-related genes; DG: depression-related genes; FR: functional relationship; IG: insomnia-related genes; UID: Uniprot ID; CCID: chemical composition ID; CC: chemical composition; TP: target protein.

\section{Declarations}

\section{Acknowledgements}

Not applicable.

\section{Authors' contributions}

WH and HXC conceived the project. WH and TXM collected the data. WH and TXM. analyzed the data. WH wrote the manuscript of the paper. WY reviewed and approved the final draft of the paper. All authors read and approved the fnal manuscript.

\section{Funding}


This work was financially supported by the National Natural Science Foundation of China (Nos. 81673697 and 81973495$)$

\section{Availability of data and materials}

The datasets used and/or analyzed during the current study are available from the corresponding author upon reasonable request.

\section{Ethics approval and consent to participate}

Not applicable.

\section{Consent for publication}

Not applicable.

\section{Competing interests}

These authors declare that there are no conficts of interest regarding this work.

\section{Author details}

${ }^{1}$ Department of Traditional Chinese Medicine Informatics, School of Chinese Materia Medica, Beijing University of Chinese Medicine, Beijing 102488, China

\section{References}

1. Yang J, Wang S, Ao R. Clinical study on the treatment of depression with Jieyu Pill. China's Naturop. 2015;23:53.

2. Shen Z, Zhu M, Zhao A. Comparative observation on efficacy of jieyu pill and maprotiline in treating depression [Internet]. Chinese J. Integr. Tradit. West. Med. 5th Hospital of Tangshan City, Hebei Province 063004. zhenmingshen@163.com; 2004. p. 415-7. Available from: https://pubmed.ncbi.nlm.nih.gov/15199625

3. Yu H. The therapeutic observation on Anxiety-related insomnia of Jie-yu pill combined with Mirtazapine pill. Hebei Med Univ. Hebei; 2004;

4. Qian H. Effectiveness of Jieyu pill in treating menopausal depression in 64 cases. Forum Tradit Chinese Med. 2017;32:42.

5. Tsuno N, Besset A, Ritchie K. Sleep and depression. [Internet]. J. Clin. Psychiatry. E0361 Epidemiology of Nervous System Pathologies, Institut National de la Santé et de la Recherche Médicale (INSERM), Hôpital La Colombière, Montpellier, France. Nrtsuno@aol.com; 2005. p. 1254-69. Available from: https://pubmed.ncbi.nlm.nih.gov/16259539

6. Manber R, Chambers AS. Insomnia and depression: a multifaceted interplay. [Internet]. Curr. Psychiatry Rep. Department of Psychiatry and Behavioral Sciences, Stanford University, 401 Quarry 
Road, Stanford, CA 94305, USA. rmanber@stanford.edu; 2009. p. 437-42. Available from: https://pubmed.ncbi.nlm.nih.gov/19909664

7. Xu Z. Clinical features of menopausal depression and discussion of nursing strategies. Electron $\mathrm{J}$ Pract Gynecol Endocrinol. 2020;7:181.

8. Guo Y, Wang Y. Research Overview and Treatment Progress of Perimenopausal Depression. J Pract Tradit Chinese Intern Med. 2020;34:67-9.

9. Xu X, Dai Y, Liu Q. Cell Communication and its pharmacology of on Traditional Chinese Medicine. World Sci Technol Tradit Chinese Med Mater Medica. 2008;1:97-102+34.

10. Jiang H, Gu W. Design and Optimization of Medicated Diet's Diagram Database Based on Neo4j. J Minzu Univ China(Natural Sci Ed. 2019;28:48-55.

11. Zhao K, Wang H, Shi N, Sa Z, Xu X. Study and Implementation on Knowledge Graph of Guizhi Decoction Associated Formulas Based on Neo4j. World Chinese Med. 2019;14:2636-2639+2646.

12. Chen J, Huang Y, Cao G. Research and Implementation of Visualization of Judicial Cases Based on Knowledge Mapping. J Hubei Univ Technol. 2019;34:72-7.

13. Feng L. Building a knowledge map of poetry in secondary school languages based on the Neo4j graph database. Shaanxi Norm Univ. 2019;

14. Liu L. Research of Automated Diagnosis based on Medical Knowledge Graph. Hunan Univ. 2018;

15. Hamosh A. Online Mendelian Inheritance in Man (OMIM), a knowledgebase of human genes and genetic disorders. Nucleic Acids Res [Internet]. ahamosh@jhmi.edu; 2004;33:D514-7. Available from: https://pubmed.ncbi.nlm.nih.gov/15608251

16. Wishart DS, Feunang YD, Guo AC, Lo EJ, Marcu A, Grant JR, et al. DrugBank 5.0: a major update to the DrugBank database for 2018. [Internet]. Nucleic Acids Res. Department of Biological Sciences, University of Alberta, Edmonton, AB T6G 2E9, Canada.; 2018. p. D1074-82. Available from: https://pubmed.ncbi.nlm.nih.gov/29126136

17. Li YH, Yu CY, Li XX, Zhang P, Tang J, Yang Q, et al. Therapeutic target database update 2018: enriched resource for facilitating bench-to-clinic research of targeted therapeutics. [Internet]. Nucleic Acids Res. Bioinformatics and Drug Design Group, Department of Pharmacy and Center for Computational Science and Engineering, National University of Singapore, Singapore 117543, Singapore.; 2018. p. D1121-7. Available from: https://pubmed.ncbi.nlm.nih.gov/29140520

18. Ru J, Li P, Wang J, Zhou W, Li B, Huang C, et al. TCMSP: a database of systems pharmacology for drug discovery from herbal medicines. [Internet]. J. Cheminform. Center for Bioinformatics, College of Life Science, Northwest A\&F University, Yangling, Shaanxi 712100, China.; 2014. p. 13. Available from: https://pubmed.ncbi.nlm.nih.gov/24735618

19. Ru J. Construction and Utilization of Traditional Chinese Medicine Systems Pharmacology Database and Analysis Platform. Northwest Agric For Univ Sci Technol. 2015;

20. Szklarczyk D, Santos A, von Mering C, Jensen LJ, Bork P, Kuhn M. STITCH 5: augmenting proteinchemical interaction networks with tissue and affinity data. [Internet]. Nucleic Acids Res. Institute of Molecular Life Sciences, University of Zurich and Swiss Institute of Bioinformatics, 
Winterthurerstrasse 190, 8057 Zurich, Switzerland.; 2016. p. D380-4. Available from: https://pubmed.ncbi.nlm.nih.gov/26590256

21. Hopkins AL. Network pharmacology: the next paradigm in drug discovery [Internet]. Nat. Chem. Biol. Division of Biological Chemistry and Drug Discovery, College of Life Science, University of Dundee, Dundee, UK. a.hopkins@dundee.ac.uk; 2008.p. 682-90. Available from: https://pubmed.ncbi.nlm.nih.gov/18936753

22. Hopkins AL, Groom CR, Alex A. Ligand efficiency: a useful metric for lead selection [Internet]. Drug Discov. Today. 2004. p. 430-1. Available from: https://pubmed.ncbi.nlm.nih.gov/15109945

23. Zhang B, Li J, Gu H, Li Q, Zhang Q, Zhang T, et al. Using Bioinformatics Approach to Explore the Pharmacological Mechanisms of Multiple Ingredients in Shuang-Huang-Lian. Sci World J [Internet]. School of Chinese Materia Medica, Beijing University of Chinese Medicine, Beijing 100102, China.; 2015; Available from: https://pubmed.ncbi.nlm.nih.gov/26495421

24. Du L, Ye X, Li M, Wang H, Zhang B, Zheng R, et al. ScienceDirect Mechanisms of traditional Chinese medicines in the treatment of allergic rhinitis using a network biology approach. 2016;1-9.

25. Zhang Q, Du R, Hou X, Lv Z, Jia C, Wang Y. ScienceDirect Analysis of new therapeutic strategies for diabetes mellitus based on traditional Chinese medicine " xiaoke" formulae. J Tradit Chinese Med Sci [Internet]. Elsevier Ltd; 2018;5:361-9. Available from: https://doi.org/10.1016/j.jtcms.2018.11.005.

26. Szklarczyk D, Gable AL, Lyon D, Junge A, Wyder S, Huerta-Cepas J, et al. STRING v11: protein-protein association networks with increased coverage, supporting functional discovery in genome-wide experimental datasets. [Internet]. Nucleic Acids Res. Institute of Molecular Life Sciences and Swiss Institute of Bioinformatics, University of Zurich, 8057 Zurich, Switzerland.; 2019. p. D607-13. Available from: https://pubmed.ncbi.nlm.nih.gov/30476243

27. Ramilowski JA, Goldberg T, Harshbarger J, Kloppmann E, Lizio M, Satagopam VP, et al. Corrigendum: A draft network of ligand-receptor-mediated multicellular signalling in human. [Internet]. Nat. Commun. 2016. p. 10706. Available from: https://pubmed.ncbi.nlm.nih.gov/26862012

28. Pontén F, Schwenk JM, Asplund A, Edqvist P-HD. The Human Protein Atlas as a proteomic resource for biomarker discovery. [Internet]. J. Intern. Med. Department of Genetics and Pathology, Uppsala University, Uppsala, Sweden. Fredrik.ponten@igp.uu.se; 2011. p. 428-46. Available from: https://pubmed.ncbi.nlm.nih.gov/21752111

29. Wang Y. Entity grammar systems: a grammatical tool for studying the hierarchical structures of biological systems. [Internet]. Bull. Math. Biol. National Laboratory of Protein Engineering, Peking University, Beijing 100871, China. yonwangpku@yahoo.com.cn; 2004. p. 447-71. Available from: https://pubmed.ncbi.nlm.nih.gov/15006443

30. Hou X, Cheng S, Ren X, Li M, Wang Y. Analysis of Pharmacological Mechanism of Jieyu Pill Based on Entity Grammar System. Mod Chinese Med. 2020;

31. Kano M, Fukudo S, Tashiro A, Utsumi A, Tamura D, Itoh M, et al. Decreased histamine H1 receptor binding in the brain of depressed patients. [Internet]. Eur. J. Neurosci. Department of Behavioural 
Medicine, Tohoku University School of Medicine, Sendai 980-8575, Japan.; 2004. p. 803-10. Available from: https://pubmed.ncbi.nlm.nih.gov/15255990

32. Giuliani P, Romano S, Ballerini P, Ciccarelli R, Petragnani N, Cicchitti S, et al. Protective activity of guanosine in an in vitro model of Parkinson's disease. [Internet]. Panminerva Med. Department of Experimental and Clinical Sciences, University of Chieti-Pescara, Chieti, Italy.; 2012. p. 43-51. Available from: https://pubmed.ncbi.nlm.nih.gov/23241934

33. Rilstone JJ, Alkhater RA, Minassian BA. Brain dopamine-serotonin vesicular transport disease and its treatment. [Internet]. N. Engl. J. Med. Institute of Medical Science, University of Toronto, Toronto, ON, Canada.; 2013. p. 543-50. Available from: https://pubmed.ncbi.nlm.nih.gov/23363473

34. Chehab FF, Lim ME, Lu R. Correction of the sterility defect in homozygous obese female mice by treatment with the human recombinant leptin. [Internet]. Nat. Genet. Department of Laboratory Medicine, University of California, San Francisco, 94143-0134, USA.; 1996. p. 318-20. Available from: https://pubmed.ncbi.nlm.nih.gov/8589726

35. Zhao Y, Sun R, You L, Gao C, Tian Z. Expression of leptin receptors and response to leptin stimulation of human natural killer cell lines. [Internet]. Biochem. Biophys. Res. Commun. School of Life Sciences, University of Science and Technology of China, 443 Huangshan Road, Hefei City, Anhui 230027, China.; 2003. p. 247-52. Available from: https://pubmed.ncbi.nlm.nih.gov/12504075.

36. Krauss M, Kinuta M, Wenk MR, De Camilli P, Takei K, Haucke V. ARF6 stimulates clathrin/AP-2 recruitment to synaptic membranes by activating phosphatidylinositol phosphate kinase type Igamma. [Internet]. J. Cell Biol. Zentrum für Biochemie und Molekulare Zellbiologie, Dept. of Biochemistry II, University of Göttingen, Humboldtallee 23, Göttingen D-37073, Germany.; 2003. p. 113-24. Available from: https://pubmed.ncbi.nlm.nih.gov/12847086

\section{Figures}

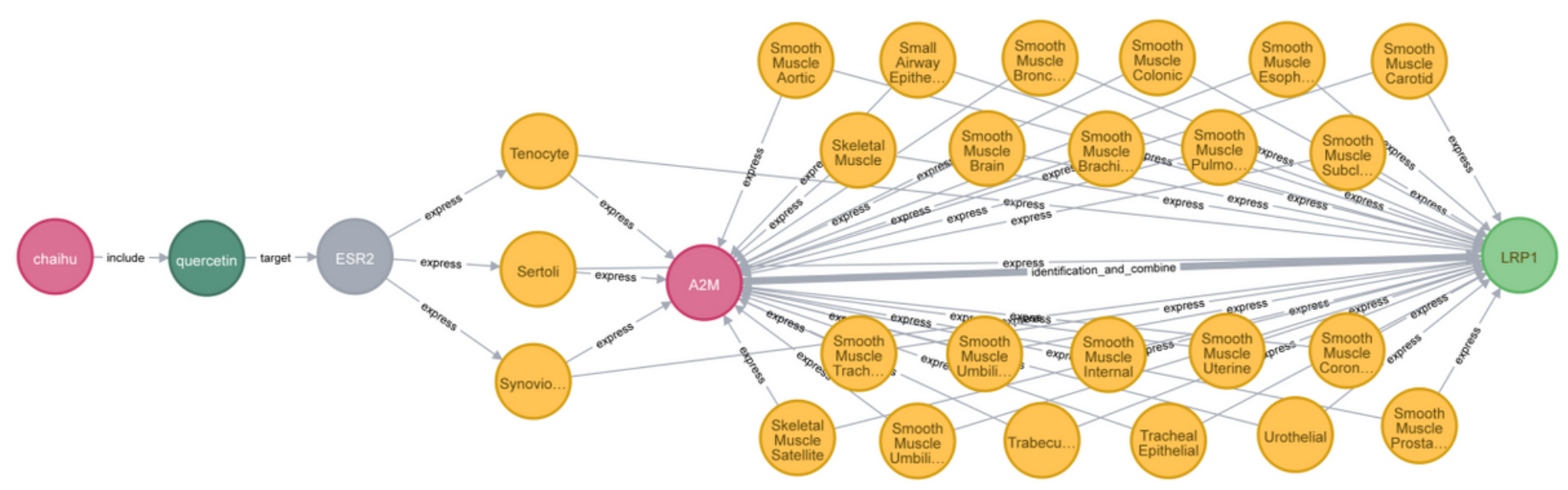

\section{Figure 1}

Partial delivery path of quercetin's bioactivity in cellular communication networks 


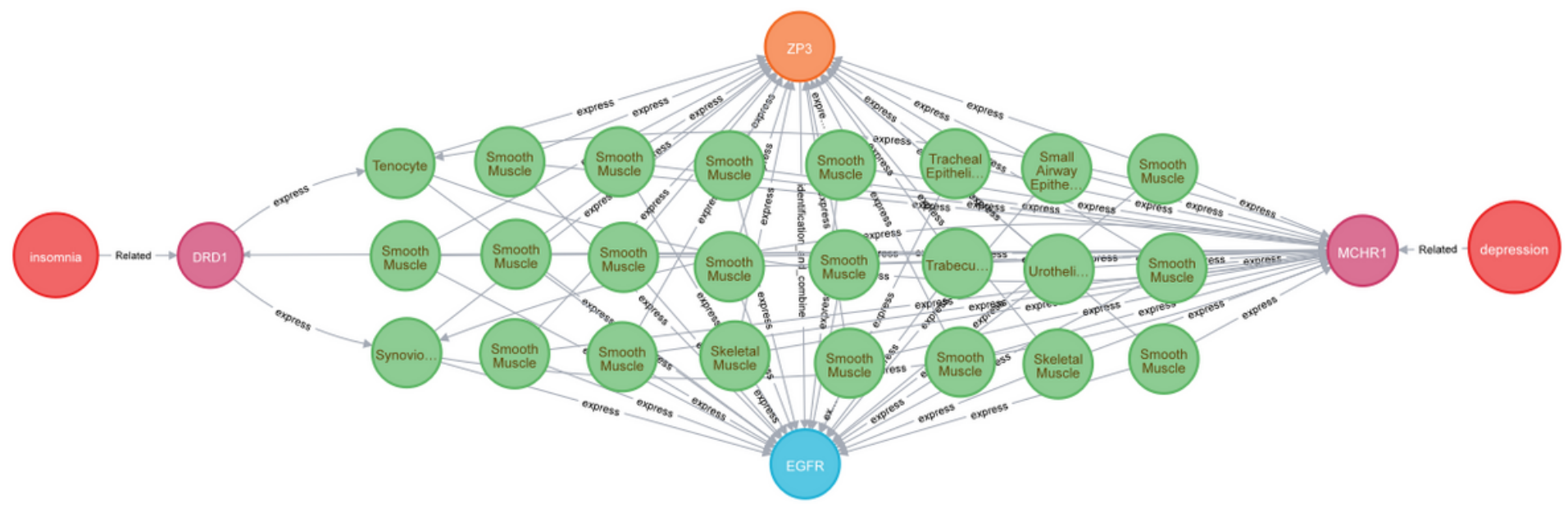

Figure 2

Relationship between insomnia and depression

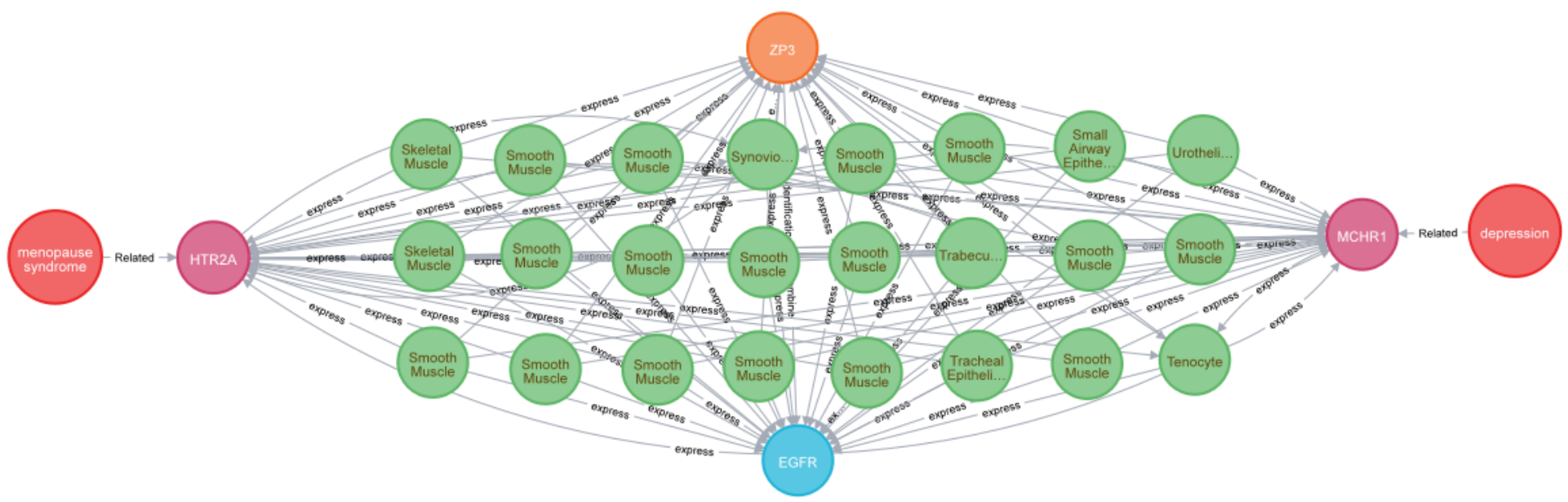

Figure 3

Relationship between menopausal syndrome and depression

\section{Supplementary Files}

This is a list of supplementary files associated with this preprint. Click to download.

- Additionalfile.docx 